\title{
Identification of Xinyang Maojian Tea Taste Using Electronic Tongue
}

\author{
Hongmei Zhang, Guangyu Zou, Xinping Liu, Yanzhong Xiao, and Wanzhang Wang \\ College of Mechanical and Electrical Engineering, Henan Agricultural University, Zhengzhou 450002, China
}

(Received December 21, 2018; accepted March 6, 2019)

Keywords: Xinyang Maojian tea, aftertaste, principal component analysis, linear discriminant analysis, support vector machine

The taste of Xinyang Maojian tea was tested using an electronic tongue to conduct a correlation analysis between the taste and quality of tea. It was determined that the astringent aftertaste had a significant relationship with the quality of tea $(p<0.01)$. The aftertaste properties of tea were evaluated before and after adding bitter, astringent, and rich (fresh aftertaste) aftertastes to six types of tea, and compared by principal component analysis (PCA) and linear discriminant analysis (LDA). PCA and LDA results were affected by the aftertaste attributes of tea soup. After the addition of aftertaste, the classification effect of PCA was reduced, and that of LDA was improved. After introducing aftertaste, LDA had the best effect on tea differentiation. Finally, a support vector machine (SVM) model was established for tea grade evaluation based on the tea aftertaste and basic taste attributes. The values of the SVM penalty function $c$ and kernel function search radius $g$ were optimized by the grid search and particle swarm optimization (PSO) methods, respectively, to compare the classification performance characteristics of the two optimization methods. The grid search method was used to analyze the global classification effect, which had a heavy calculation burden and was unable to achieve further optimization near the optimum point. The PSO method had iterative characteristics and it was easier to determine the optimal point. The classification accuracies of the training and test sets of the grid search method were 86.11 and $87.5 \%$, respectively, and those of the training and test sets of the PSO method both reached $100 \%$, indicating the superiority of the PSO method over the grid search method. In conclusion, the effectiveness of an electronic tongue for distinguishing the quality of Xinyang Maojian tea was demonstrated.

\section{Introduction}

Xinyang Maojian tea is one of the top ten most famous teas in China. ${ }^{(1)}$ It is popular among people because of its unique taste. Xinyang Maojian tea is rich in polyphenols, caffeine, and amino acids, which are important components that confer tea flavor diversity. ${ }^{(2)}$ However, it is often difficult to identify subtle changes in these components without professional training. Therefore, a scientific evaluation method based on instrument evaluation analysis is needed.

*Corresponding author: e-mail: hmzh86022625@sina.com

https://doi.org/10.18494/SAM.2019.2244 
At present, the main methods of instrument-based detection used to analyze tea are gas chromatography and mass spectrometry, near-infrared spectroscopy, chemical composition detection, electronic tongue analysis, electronic nose analysis, and so forth. ${ }^{(3-7)}$ The taste of tea soup is an important standard for evaluating tea quality. To explore the relationship between the taste and quality of Xinyang Maojian tea, an electronic tongue was used to analyze the taste of Xinyang Maojian tea.

An electronic tongue is an intelligent electronic recognition system that imitates the human taste mechanism and is widely used in food detection. ${ }^{(8)}$ Liu et al. used an electronic tongue to detect the taste of beer, and the error rate of prediction of sensory evaluation information by a fuzzy neural network was between 0.0048 and $0.0993 .{ }^{(9)}$ Rudnitskaya et al. used a potentiometric electronic tongue to detect wine maceration with oak chips and found that the electronic tongue was capable of quantifying total oak, total phenolics, main phenolic fractions, and the concentration of oak chips. ${ }^{(10)}$ The electronic tongue has the advantages of being rapid, simple, reliable, and highly sensitive. ${ }^{(11)}$ It is suitable for the detection of tea taste and has been used in the evaluation of some black tea and green tea. ${ }^{(12-14)}$

The quality of Xinyang Maojian tea is affected by not only its chemical composition, aroma, and taste but also its aftertaste. The taste and aftertaste of Xinyang Maojian tea are mainly bitter and astringent, respectively. In this study, six different grades of Xinyang Maojian tea were brewed, and the taste of tea soup was detected using an electronic tongue. Principal component analysis (PCA) and linear discriminant analysis (LDA) were used to compare the effects of aftertaste attributes on tea quality. Finally, the support vector machine (SVM) model was applied to the analysis of aftertaste factors. The SVM model is optimized by the grid search and particle swarm algorithm to select the model with the best classification effect. This study provides a theoretical basis for recognizing the quality of Xinyang Maojian tea through tea taste detection.

\section{Materials and Methods}

\subsection{Materials}

The Xinyang Maojian tea samples were picked before the Qingming Festival in the Xinyang district, Henan Province in April 2018. Six grades of tea were used in the experiment. The grades of tea were labeled T1, T2, T3, T4, T5, and T6 with T1 indicating the highest grade and T6 the lowest. The classification of tea grades was determined as described below. The tea that consisted of entirely one bud and one leaf is the first grade of tea. One bud and one (or two) leaf/leaves that accounted for more than $90 \%$ of the tea is the second grade of tea. One bud and one (or two or three) leaf/leaves that accounted for more than $85 \%$ of the tea is the third grade of tea. One bud and one (or two and three) leaf/leaves that accounted for more than $75 \%$ of the tea is the fourth grade of tea. One bud and two (or three) leaves that accounted for more than $70 \%$ of the tea is the fifth grade of tea. One bud and two (or three) leaves that accounted for more than $65 \%$ of the tea is the sixth grade of tea. All grades of tea were stored in tin foil bags. Before the experiment, the tea was refrigerated at $-4^{\circ} \mathrm{C}$. 


\subsection{Electronic tongue}

In this study, we used the Taste-Sensing System SA402B, which is manufactured by the Intelligent Sensor Technology Co., Ltd., Japan. The electronic tongue is mainly composed of three parts: the detection instrument, sensor array, and operating computer. The sensor array is composed of five taste sensors and two reference electrodes. When the electronic tongue works, it collects the change in the signal of the film potential of the sensor. The electronic tongue's software automatically converts the measured potential into a taste value. The electronic tongue can not only detect the five basic tastes of sour, bitter, astringent, umami, and salty but can also analyze the bitter, astringent, and fresh (rich) aftertastes. The five tastes of sour, bitter, astringent, umami, and salty can be detected by a sourness sensor (CA0), a bitterness sensor (C00), an astringency sensor (AE1), an umami sensor (AAE), and a saltiness sensor (CT0), respectively. The CA0 sensor can identify citric or acetic acid in samples. The C00 sensor often responds well to acidic bitter substances. The AE1 sensor can detect some astringent substances. The AAE sensor can identify the amino acid or nucleic acid in samples, and the $\mathrm{CT} 0$ sensor can identify salts in samples such as $\mathrm{NaCl}$ and $\mathrm{KCl}$.

\subsection{Measurement}

The tea brewing method is as follows: $5.00 \mathrm{~g}$ of tea leaves was added to a teapot. Then, $250 \mathrm{~mL}$ of boiling pure water was poured into the teapot, and the mixture was allowed to stand for $5 \mathrm{~min}$ at ambient temperature. After brewing, the tea leaves were filtered out to prepare the tea soup for the experiment.

The mechanical arm of the electronic tongue was automatically controlled by computer software. Taste sensors and reference electrodes were installed on the mechanical arm. The sensors were cleaned in positive and negative cleaning solutions for $90 \mathrm{~s}$ and in the first two reference solutions for $120 \mathrm{~s}$. The sensors were balanced and calibrated for $30 \mathrm{~s}$, and the sample was tested for $30 \mathrm{~s}$. Then, the sensors were rapidly cleaned twice with the two reference solutions, each time for $3 \mathrm{~s}$. Next, the sensors were moved into the new reference solution to measure the aftertaste for $30 \mathrm{~s}$. Finally, the sensor was moved back into the positive and negative cleaning solutions to complete the cycle. The experiment involved 20 identical operations for each of the tea samples of six grades.

\section{Results and Discussion}

\subsection{Original data analysis and data preprocessing}

The electronic tongue detects the taste of tea mainly through its five taste sensors. The results of six different tea grades are shown in Fig. 1. Xinyang Maojian tea has the most obvious degree of distinction due to its astringency and astringent aftertaste. The relationship between tea grade and tea taste is shown in Table 1 . Tea quality positively correlated with the values of sourness, bitterness, astringency, and astringent aftertaste, and negatively correlated 


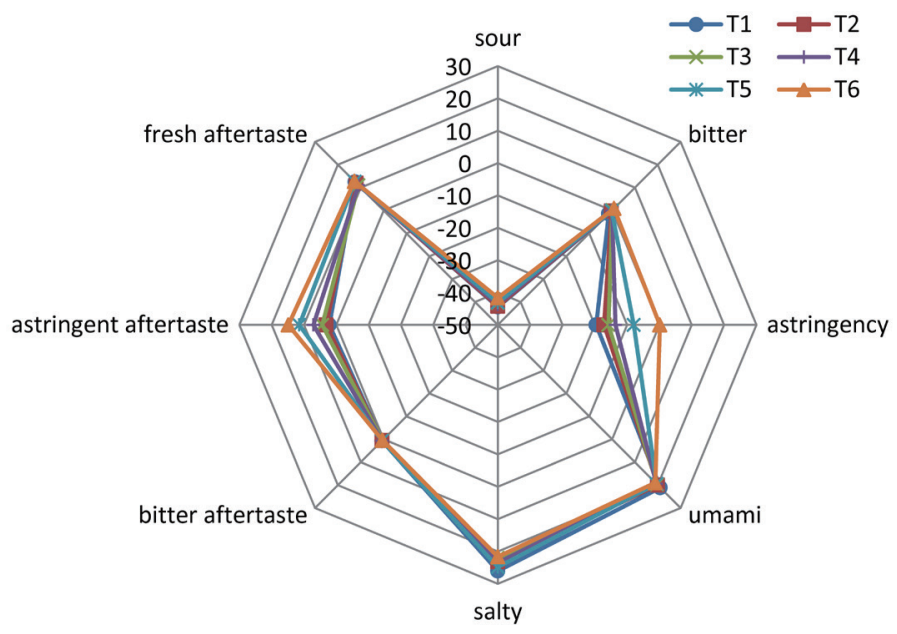

Fig. 1. (Color online) Response patterns of sensors for six different grades of Xinyang Maojian tea.

Table 1

Correlation between taste attributes and tea quality.

\begin{tabular}{lcccccccc}
\hline Tea quality & Sourness & Bitterness & Astringency & Umami & Saltiness & $\begin{array}{c}\text { Bitter } \\
\text { aftertaste }\end{array}$ & $\begin{array}{c}\text { Astringent } \\
\text { aftertaste }\end{array}$ & $\begin{array}{c}\text { Fresh } \\
\text { aftertaste }\end{array}$ \\
\hline Linear correlation & 0.898 & 0.842 & 0.944 & -0.913 & -0.492 & -0.474 & 0.968 & 0.247 \\
Significance & 0.015 & 0.035 & 0.005 & 0.011 & 0.322 & 0.342 & 0.002 & 0.637 \\
\hline
\end{tabular}

with the value of umami. The $p$ value of astringent and astringent aftertastes was less than 0.01 , indicating that these two tastes play a significant role in the evaluation of tea quality. To date, most studies on tea based on an electronic tongue have focused only on the basic taste and the cross-influence of taste, and few reports have focused on the aftertaste. However, aftertaste affects the evaluation of tea and is one of the bases for judging the quality of tea. Therefore, PCA and LDA were used in this study to discuss the effect of aftertaste. In view of the large difference between taste values, the z-score method was used to standardize the taste data of Xinyang Maojian tea. The z-score method can remove the data dimension and make the different features comparable, which enables comparison between different indices.

\subsection{PCA and LDA}

The PCA results of five taste values measured using the electronic tongue are shown in Fig. 2(a). The distinction among the six tea grades is obvious. However, the quality of tea should not only be evaluated from the original taste but also from the aftertaste. Therefore, five basic flavors were combined with bitter, astringent, and fresh aftertastes for the analysis. PCA was performed on the mixed information of basic taste and aftertaste. The results are shown in Fig. 2(b). The spacing within the tea group of grades T1, T5, and T6 increased, but the differentiation degree was still very high. The aggregation effect of grade T2 was worse. The 


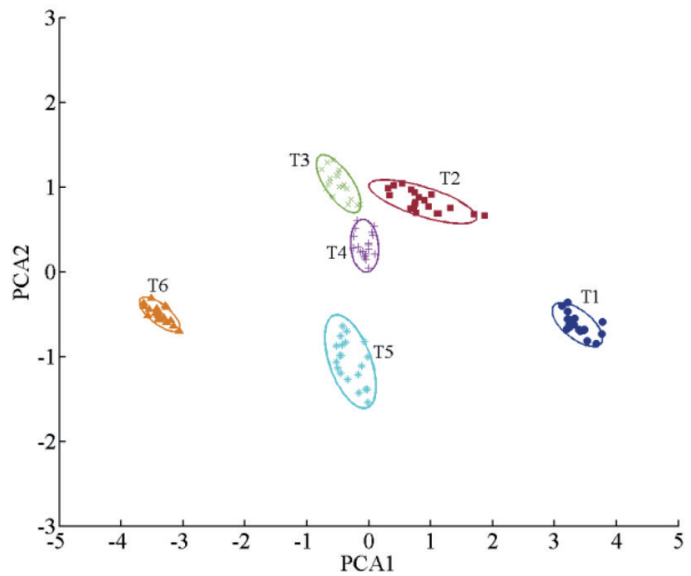

(a)

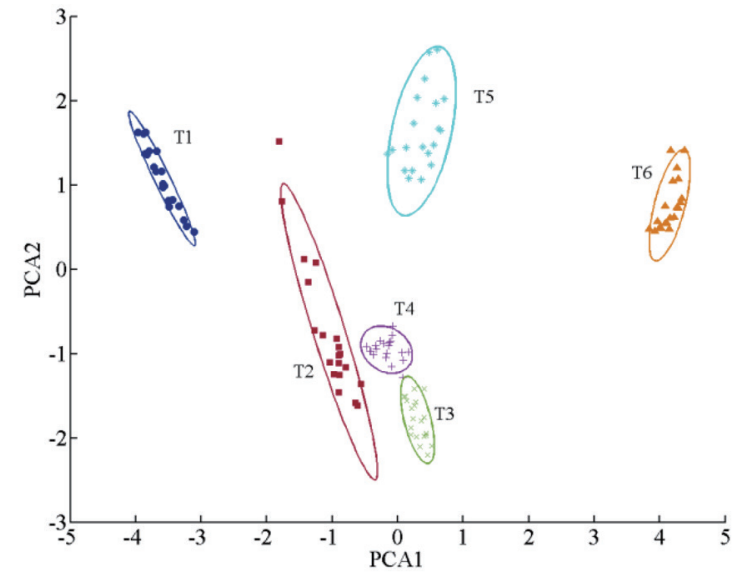

(b)

Fig. 2. (Color online) PCA plots of electronic tongue data for different qualities of tea. (a) PCA plots of the original taste. (b) PCA plots of the aftertaste.

interval between grades T2 and T4 decreased. The six grades of tea can be distinguished by PCA before and after introducing aftertaste. However, after the introduction of the aftertaste, PCA was less effective in distinguishing the tea grade than before the introduction.

The LDA results of the five taste values measured using the electronic tongue are shown in Fig. 3(a). LDA can effectively distinguish tea quality before and after the introduction of aftertaste. As shown in Fig. 3(b), the differentiation degree of tea grades T2, T3, and T4 increased significantly after the aftertaste was introduced. This result indicates that the aftertaste property of tea plays a certain role in the evaluation of tea leaves. In addition, the LDA results showed that compared with PCA, LDA could better distinguish tea leaves of different qualities.

\subsection{SVM for distinguishing tea quality}

\subsubsection{Grid search optimization algorithm}

SVM is a machine learning algorithm based on statistical learning theory. ${ }^{(15)}$ It follows the principle of structural risk minimization and has a good generalization ability for small samples. ${ }^{(16)}$ SVMs can be classified into different types according to different classification standards. In this study, C-SVC was selected for the SVM, and a linear function was selected for the kernel function. ${ }^{(17,18)}$ To improve the accuracy of the SVM, the penalty function ( $c$ value) and the kernel function search radius ( $g$ value) need to be optimized. The higher the $c$ value, the higher the accuracy of the SVM training set and the lower the fault tolerance of the SVM. However, the generalization ability of the SVM and the prediction ability of the training set become worse. The smaller the $c$ value, the better the SVM can tolerate error and the classification performance becomes worse. When the $g$ value is very large, the generalization ability of the SVM is worse. When the $g$ value is very small, the sensitivity of the data decreases. Therefore, 


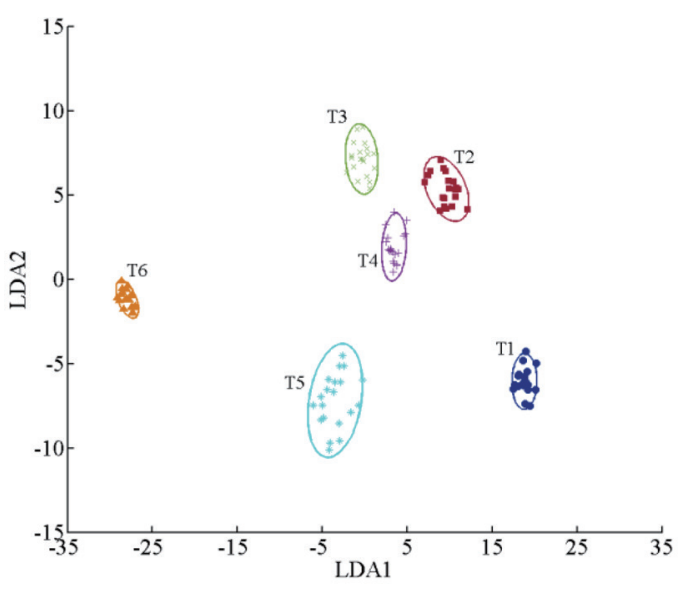

(a)

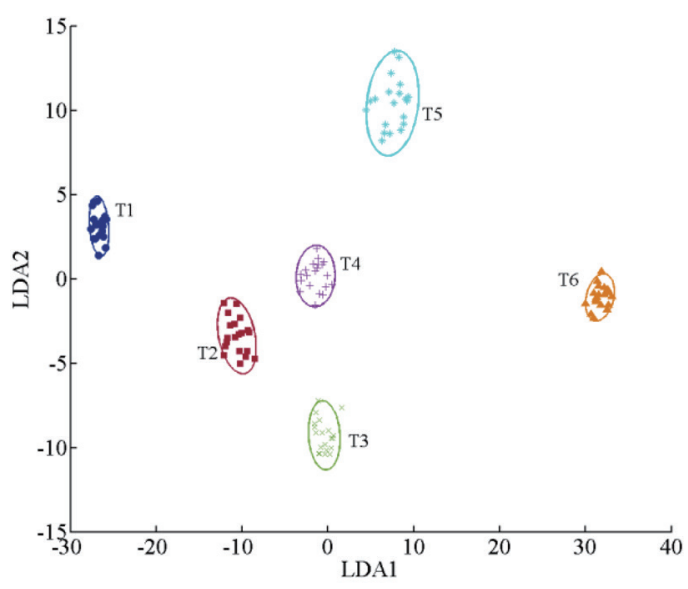

(b)

Fig. 3. (Color online) LDA plots of electronic tongue data for different qualities of tea. (a) LDA plots of the original taste. (b) LDA plots of the aftertaste.

to obtain better classification results, we need to coordinate the $c$ and $g$ value relations in the SVM. The $c$ and $g$ values can be optimized through cross-validation combined with a grid search.

Sixty percent of data for all tea leaves tested was randomly selected as the training set to build the SVM model, and the remaining $40 \%$ was used as a validation set for the verification of the training results. The optimization results of the grid search are shown in Fig. 4. The grid search method was adopted to obtain a $c$ value of 0.010 and a $g$ value of 0.226 . At this point, the accuracy rate of the training set was $86.11 \%$, and that of the verification set was $87.5 \%$. The grid search method optimization takes $c$ and $g$ value ranges as horizontal and vertical coordinates, and the spatial rectangle is divided equally by a fixed step length. The best $c$ and $g$ values were selected by comparing the accuracy of each grid classification. Theoretically, when the step length is sufficiently small, the global optimal solution can be found. However, this causes the problem of requiring large amounts of calculations and time. When the step size is increased, further optimization cannot be carried to the best advantage. It is easy to miss the $c$ and $g$ values that are more favorable for classification. Therefore, adopting a precise method of optimizing $c$ and $g$ values may make the classification effect clearer.

\subsubsection{Particle swarm optimization (PSO) algorithm}

PSO is an evolutionary method based on swarm intelligence optimization. ${ }^{(19,20)}$ On the basis of the foraging behavior of birds, PSO abstracts each bird into a particle in a certain search space. Particles have independence and sociality. That is, each particle can find its optimal position from the target through its own motion trajectory. At the same time, information exchange among particles is shared to find the optimal position of the whole particle swarm distance to the target. The velocity and position update formula of PSO is 


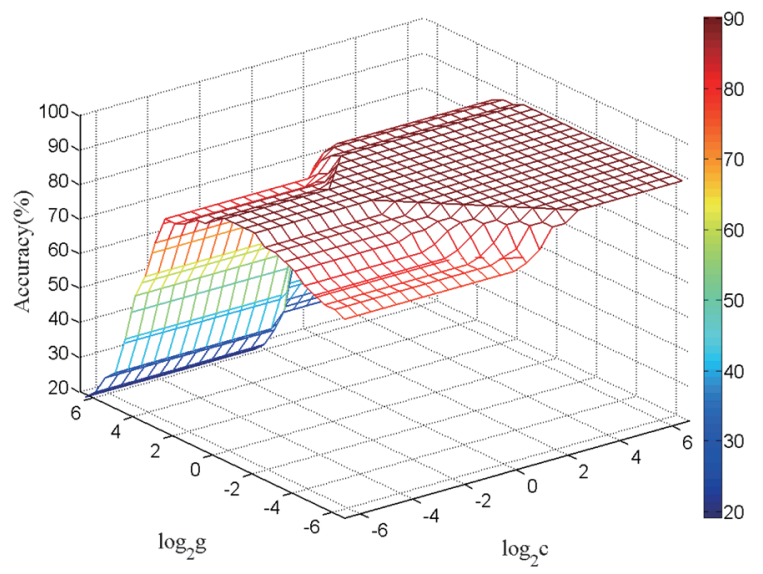

Fig. 4. (Color online) Optimization of the grid search method.

$$
\begin{gathered}
v(t+1)=w v(t)+c_{1} \operatorname{rand}()\left(p_{\text {best }}(t)-p(t)\right)+c_{2} \operatorname{rand}()\left(g_{\text {best }}(t)-p(t)\right), \\
p(t+1)=p(t)+v(t+1),
\end{gathered}
$$

where $v(t+1)$ is the velocity after particle update, $v(t)$ is the velocity before particle update, $p_{\text {best }}$ is the optimal position of the particle at time $t, g_{\text {best }}$ is the optimal location of the population at time $t, w$ is the weight of inertia $(w=1), c_{1}$ and $c_{2}$ are learning factors $\left(c_{1}=1.5, c_{2}=1.7\right), p(t+1)$ is the updated position of the particle, and $p(t)$ is the original position of the particle.

In the particle swarm algorithm, the learning factor $c_{1}$ affects the local search ability of particles, while the learning factor $c_{2}$ affects the global search ability of particles. The inertia weight $w$ determines the particle search range. The optimization of the particle group algorithm for $c$ and $g$ values was performed as follows. First, basic information, such as the $c$ search range, $g$ search range, population size, and maximum number of iterations of $c$ and $g$ values, was given. Second, 20 particles were generated randomly and given a certain initial velocity. Then, SVM learning was started, taking the error of the current position prediction of the particle as a fitness value and comparing the fitness value to find the optimal solution of the particle itself and the particle population. Finally, iterations were repeated until the preset precision or maximum iterations were achieved.

As previously stated, $60 \%$ of all tea data was randomly selected as the training set and $40 \%$ was selected as the validation set to build the SVM model. The optimal iteration results of PSO are shown in Fig. 5. The PSO algorithm was used to find $c$ and $g$ values of 0.100 and 0.242 , respectively. The classification accuracy of the optimized training and validation sets can reach $100 \%$. The trajectory of each particle in PSO is random. In Fig. 5, the particles are not necessarily able to find the optimal point through only one iteration. Therefore, we still need multiple iterations to ensure the optimal $c$ and $g$ values. PSO algorithms balance the local and global search capabilities of particles. This algorithm can prevent particles from falling into the local optimum and find the point with better classification performance in the global search. 


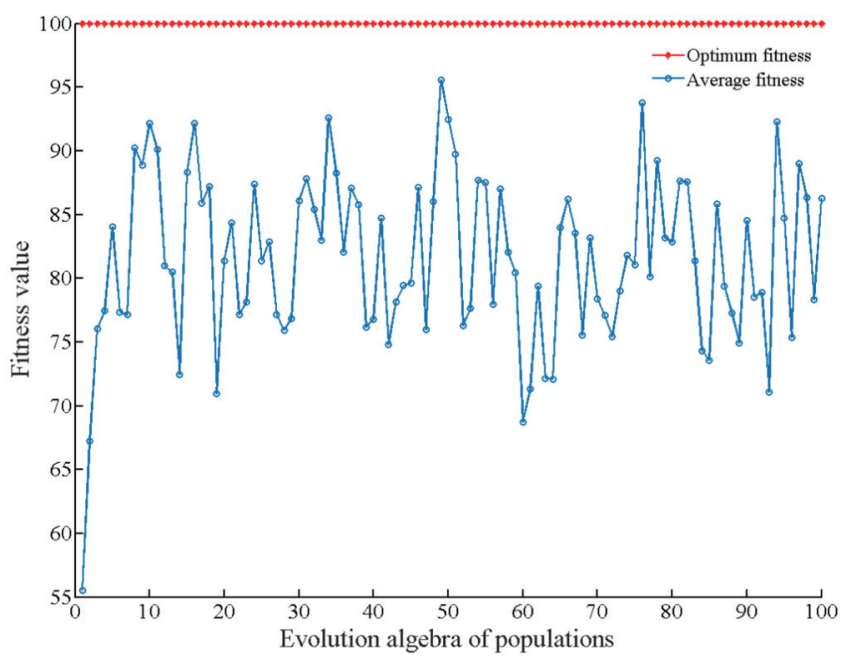

Fig. 5. (Color online) Iterative optimization of PSO.

Table 2

Comparison of the classification results of different optimization methods.

\begin{tabular}{lcccc}
\hline & $c$ value & $g$ value & Accuracy of training set & Accuracy of validation set \\
\hline Grid search method & 0.010 & 0.226 & $86.11 \%$ & $87.5 \%$ \\
PSO method & 0.100 & 0.242 & $100 \%$ & $100 \%$ \\
\hline
\end{tabular}

\subsubsection{Comparison of two optimization methods}

The classification results of the SVM algorithm based on a grid search and the SVM algorithm based on a particle swarm search are shown in Table 2. The calculation principle of the grid search method is relatively simple. However, the results of the rough search are not sufficiently accurate, and the accuracy rate of the data sets for training and testing is less than $90 \%$. The PSO algorithm has a stronger local search ability than the grid search optimization. The PSO algorithm requires repeated iterations to complete optimization, and the calculation time is longer than the grid search method. However, both algorithms can be completed in a few seconds. PSO can be further optimized near the optimal point for better advantages, which improves the accuracy of the classification results and can find $c$ and $g$ values with better classification effects. After the optimization of PSO, the accuracy rate of the training and validation sets can reach $100 \%$.

\section{Conclusions}

In this study, an electronic tongue was used to detect and analyze the taste of Xinyang Maojian tea. A method of tea quality detection based on electronic tongue technology was presented. The results showed that the differences in astringency and astringent aftertaste were most pronounced among different grades of tea. The sour, bitter, astringent, and astringent 
aftertaste values of tea increased with tea grade, while the fresh taste value decreased with tea grade. The results of PCA and LDA indicate that the taste information of tea can be divided into six grades. After combining the tea aftertaste information, the PCA classification effect decreased, whereas the LDA classification effect improved. In addition, the LDA classification effect was better. Information on tea aftertaste is one of the factors influencing tea quality evaluation. The SVM model based on basic taste and aftertaste information can be used to evaluate tea quality. The accuracies of the training and validation sets of the SVM model obtained by the grid search method are 86.11 and $87.5 \%$, respectively. The accuracies of the training and validation sets of the SVM model obtained by the PSO method are both $100 \%$. PSO is better than grid search, with an optimal $c$ value of 0.1 and a $g$ value of 0.242 . This method based on an electronic tongue is effective and provides a referential recognition method for the evaluation of tea quality.

\section{Acknowledgments}

This work was financially supported by the Chinese National Foundation of Nature and Science through Project 31501213, the special Fund for Henan Agriculture Research System through Project S2015-02-G07, the Fund for Youth Teachers of Universities of Henan Province through Project 2015GGJS-077, and the National Agricultural Industry Technology System through Project CARS-03).

\section{References}

1 Q. P. Zhang, S. W. Nie, Y. T. Zhang, D. M. Liu, T. K. Li, C. L. Kou, Q. P. Zou, L. Z. Lv, and L. J. Zhao: Southwest Chin. J. Agric. Sci. 28 (2015) 94.

2 R. M. Wu, J. W. Zhao, Q. S. Chen, and X. Y. Huang: Trans. CSAE 27 (2011) 378.

3 F. He, Y. R. L. Qian, and M. C. Qian: Food Chem. 239 (2017) 622.

4 F. Zhao, H. T. Lin, N. X. Ye, and J. P. Yu: Trans. CSAE 30 (2014) 269.

5 P. Yu, AS. Yeo, M. Y. Low, and W. Zhou: Food Chem. 155 (2014) 9.

6 M. Scampicchio, S. Benedetti, B. Brunetti, and S. Mannino: Electroanalysis 18 (2010) 1643.

7 L. Gao, Z. H. Gao, W. T. Zhao, X. S. Hu, and J. H. Wu: J. Chin. Inst. Food Sci. Technol. 17 (2017) 206.

8 S. Jiang, Q. Q. Jiang, X. F. Hu, Y. Yang, and Y. Y. Ni: Trans. CSAE 25 (2009) 345.

9 J. J. Liu, J. L. Yang, W. Wang, S. L. Fu, Y. Shi, and H. Men: Sens. Mater. 28 (2016) 785.

10 A. Rudnitskaya, L. M. Schmidtke, A. Reis, M. R. Domingues, I. Delgadillo, B. Debus, D. Kirsanov, and A. Legin: Food Chem. 229 (2017) 20.

11 D. Chen, T. Ma, W. L. San, C. Wang, and Q. H. Li: Food Sci. 38 (2017) 168.

12 W. He, X. Hu, L. Zhao, X. Liao, Y Zhang, M. W. Zhang, and J. H. Wu: Food. Res. Int. 42 (2009) 1462.

13 Q. Chen, J. Zhao, and S. Vittayapadung: Food. Res. Int. 41 (2008) 500.

14 P. Saha, S. Ghorai, B. Tudu, R. Bandyopadhyay, and N. Bhattacharyya: IEEE Trans. Instrum. Meas. 63 (2014) 2472.

15 L. L. Wu, D. Zheng, B. Z. Zheng, A. Y. Lin, and J. B. Pan: Chinese J. Sens. Actuators 26 (2013) 1473.

16 S. Qiu, L. Gao, and J. Wang: J. Food Eng. 144 (2015) 77.

17 S. M. Cheng, J. Wang, Y. H. Ma, Y. W. Wang, and Z. B. Wei: Trans. CSAE 27 (2011) 132.

18 S. M. Cheng, Y. H. Ma, B. Zhou, Y. W. Wang, and J. Wang: Chin. J. Sens. Actuators 24 (2011) 941.

19 S. U. Khan, S. Yang, L. Wang, and L. Liu: IEEE Trans. Mag. 52 (2016) 1.

20 E. C. Bezerra, R. P. S. Leão, and A. P. D. S. Braga: J. Control. Autom. Electrical Syst. 28 (2017) 785. 


\section{About the Authors}

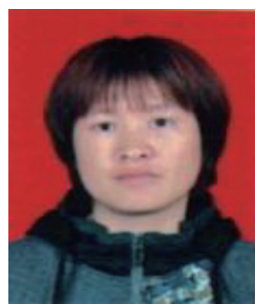

Hongmei Zhang received her B.E. and M.S. degrees from Henan Agricultural University, China, in 1999 and 2004, respectively, and her Ph.D. degree from the zhejiang University, China, in 2007. From 2007 to 2010, she was a lecturer at Henan Agricultural University, China. Since 2010, she has been an assistant professor at Henan Agricultural University. Her research interests are in nondestructive testing technology.

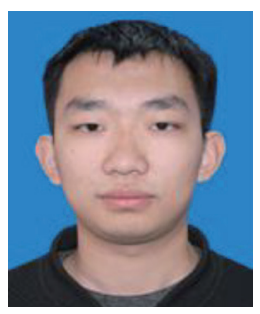

Guangyu Zou received his B.E. degree from Henan Agricultural University, China, in 2016. In 2016, he started studying for his master's degree in Henan Agricultural University. His research interest is in the intelligent detection of agricultural products.

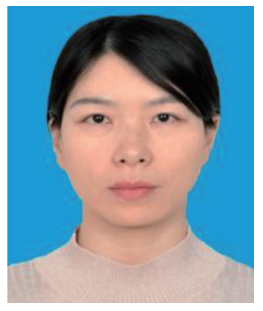

Xinping Liu received her B.E. and M.S. degrees from Henan Agricultural University, China, in 2000 and 2017, respectively. Since 2006, she has been a laboratory assistant at Henan Agricultural University. Her research interests are in nondestructive testing technology.

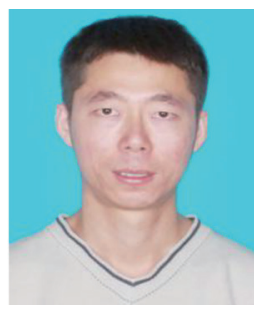

Yanzhong Xiao received his B.E degree from SIAS University, China, in 2016. Since 2017, he has been studying for a master's degree in Henan Agricultural University. His research interest is in maize plot breeding and yield measurement systems.

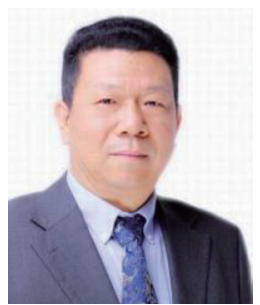

Wanzhang Wang received his B.E. degree from Henan Agricultural University, China, in 1985 and his Ph.D. degrees from the South China Agricultural University, China, in 2005. Since 2005, he has been a professor at Henan Agricultural University. His research interest is in intelligent agricultural equipment. 